“드 2014 IEEE. Personal use of this material is permitted. Permission from IEEE must be obtained for all other uses, in any current or future media, including reprinting/republishing this material for advertising or promotional purposes, creating new collective works, for resale or redistribution to servers or lists, or reuse of any copyrighted component of this work in other works." 


\title{
An implementation of the path integrator mechanism of head direction cells for bio-mimetic navigation.
}

\author{
Ankur Sinha and Jack Wang
}

\begin{abstract}
Head direction cells are thought to be an integral part of the neural navigation system. These cells track the agent's current head direction irrespective of the host's location. In doing so, they process a combination of inputs: angular velocity and visual inputs are major effectors; to correctly encode the agent's current heading. There are close to fifteen models of head direction cell systems found in literature today. Very few of these models have been implemented for biomimetic navigation in robots. In this paper, we describe an implementation of the head direction cell system on the ROS robotic platform as a first step towards a bio-mimetic navigation system for the PR2 robot.
\end{abstract}

\section{INTRODUCTION}

$\mathbf{N}$ AVIGATION is a necessary capability any animal must possess to survive. Similarly, it is also a capability that must be implemented while designing a robotic system capable of carrying out any meaningful tasks. It is well known that even smaller mammals, such as rats, have sufficiently well developed navigation systems that enable them to carry out tasks necessary for their survival, such as foraging for food or finding shelter. It is, therefore, of great interest to study these biological navigation systems and attempt to implement bio-mimetic navigation systems on robots.

Biological navigation is quite different from classical robotic navigation. Classical robotic navigation stems from nautical navigation[1] and attempts to track the precise spatial parameters of the agent at all times. Biological navigation, on the other hand, does not always follow the same localization and planning methods. Trullier et al.[2] and later Franz \& Mallot[1], reviewed biological navigation in detail and documented that the methods used in biological navigation are goal oriented. Some levels of biological navigation, for instance, do not require the animal to be aware of its precise location or global heading. The animal navigates purely with reference to a prominent landmark in its environment, for example. Trullier, Franz, Mallot and their colleagues categorized biological navigation into behavioural levels on the basis of the information required in navigation tasks. Their reviews, however, studied biological navigation at a high, behavioural level. At a much lower level, the neural processes that make navigation in biology possible form a vast area of research in themselves.

Discoveries of neurons that provide information on the agent's spatial parameters, such as head direction cells[3],

Ankur Sinha and Jack Wang are with the Faculty of Engineering and Information Technology, The University of Technology, Sydney, Australia (email: ankur.sinha@student.uts.edu.au,jianguo.wang@uts.edu.au).

We're most grateful to Dr. Xun Wang at the "Magic" Lab, The University of Technology, for his inputs and assistance with the PR2 robot.
[4], place cells [5] and grid cells[6], have lead to the formation of a cognitive map theory[7], [8], [9], [10], [11], [12] of biological navigation. This theory states that via a combination of neurons with specific behaviours, animals maintain a "cognitive map" of their environment.

The information contained by head direction, place and grid cells together is equivalent to the spatial parameters that are kept track of in classical robotics: position, heading and velocity. Head direction cells encode the agent's current head direction in the horizontal plane irrespective of its location. Place cells, similarly, encode the agent's current location in an environment in the form of place fields. Both these neuron sets have path integrator components that integrate angular and translational velocities respectively to calculate the agent's current head direction, like a biological INS[13]. Similar to head direction and place cells, grid cells also encode spatial information about the agent by encoding a representation of Euclidean space.

Head direction cells are capable of contributing to two types of navigation systems. The first, as mentioned, is the path integrator system where angular velocity is integrated to encode the current heading of the animal. The second, landmark navigation, is borne out the association of their preferred directions to prominent visual cues in the environment. In this paper, we detail an implementation of a model of the path integrator mechanics of head direction cell sets, as proposed by Stringer et al.[14], on the ROS robotic platform[15] as a starting point towards a head, place and grid cell based bio-neuro-mimetic navigation system. We also cover some extensions that we've made to Stringer et al.'s model that will enable head direction cells to associate with visual cues in the future.

In section I-A, we provide an introduction to head direction cells and briefly discuss their computational modelling in section I-B. We then detail our model in section $\amalg$ In section III. we present our results and briefly discuss challenges and our future work plans in section IV. Finally, we summarize and conclude in section $\mathrm{V}$.

\section{A. Head direction cells}

Since their discovery by Ranck[4], head direction cells have been subjected to a great amount of research. Head direction cells are neurons that maintain a firing response that encodes the agent's current head direction. Each individual neuron in a head direction cell system fires maximally when the agent faces a particular direction. This is referred to as the preferred direction of the particular neuron. A set of such neurons, the preferred directions of which together encom- 
pass all 360 degrees, therefore, acts like an internal compass, tracking the head direction of the animal as it moves about. This section briefly covers the properties of head direction cells. For detailed information on the properties of head direction cells, we refer the reader to Taube's comprehensive reviews [16], [17], [18].

Head direction cells have been found in components of the limbic system: the postsubiculum[4], the anterior dorsal nucleus of the anterior thalamus[19], the dorsal sector of the caudal lateral dorsal thalamic nucleus[20], areas of the retrosplenial cortex[21], portions of the extra-striate cortex[21], lateral mammillary nuclei[22]. Other than these components of the limbic system, many of which are also present in the classical Papez circuit, head direction cells have also been located in the dorsal striatum[23], [24].

Cells that fire only as a function of the animals current heading have also been found in the presubiculum of monkeys[25]. These cells were not influenced by the monkey's location and were seen to be independent of the monkey's "spatial view".

Experiments in which various components of the environment were selectively modified to ascertain their effect on head direction cell firing have been undertaken in abundance. Head direction cells are found to anchor their preferred directions to salient visual landmarks: experiments where salient visual cues were temporarily removed saw the preferred directions return to their original configuration when the cues were reinstalled[26], [27], [28]. Blair and Sharp did similar experiments to ascertain how visual and vestibular cues interact [29] and observed that they, along with landmark and motor signal information, were responsible for the firing of head direction cells. In a more recent study, Zugaro et al. established that head direction cells could reorient in the presence of familiar visual landmarks in as short a time as $80 \mathrm{msec}$ [27]. Rotation of auditory cues were not found to effect the head direction cells in a similar manner[30]. Olfactory cues have been found to cause a shift in the preferred direction of head direction cells in about half of the cases. However, the shift, as with visual cues, was less than the shift of the cue itself[31]. While there a shift in preferred directions was noted when the environment was changed from cylindrical to rectangular, no shift was observed when the environment was changed from cylindrical to square[26].

The firing rate of head direction cells has been found to be proportional to both translational motion[32], [33] and angular velocity in general[19], [34], [35].

Head direction cells in the anterior dorsal nucleus have been found to exhibit anticipatory firing, in that they predicted the animal's future head direction about $25 \mathrm{msec}$ in advance[35], [36]. Time shift analysis [34] and further recordings [36] confirmed this property in the anterior dorsal nucleus. Head direction cells in the postsubiculum were not found to exhibit predictive properties.

It is noteworthy that an attractor nature is generally assumed for the head direction cell network. While this may be true for head direction cells in the postsubiculum, the absence of GABA containing interneurons which are responsible for providing inhibitory influences necessary for attractor formation in the anterior dorsal nucleus indicates otherwise in this region [37], [38].

\section{B. Computational modelling of head direction cells}

Various computational models of the head direction cell system have been proposed in literature. These models are identical in that they all attempt to replicate the biological data that is known about head direction cells: firing rate profiles, neural processing, underlying architecture.

The first attempt at an explanation of the path integrator dynamics of head direction cells was given by McNaughton et al[39]. While this was more an outline and was not computationally modelled, it laid the foundation for a processing system based on associations between sets of neurons. McNaughton and colleagues proposed that the head direction system followed a look up table system where associations were built between the current heading, angular velocity and future velocity. For each possible pair of angular velocity and head direction, a future head direction would be mapped. They proposed that such mappings could easily be set up in an artificial neural network. While such mappings can be set up, it is improbable that all values of angular velocity and head direction can be included. The proposal also did not explain how such a method would result in near Gaussian triangular firing rate profiles as seen in head direction cell systems.

Skaggs et al. replaced the look up table concept with a ring attractor network [40]. This was the first model to use local co-operation and global inhibition that would result in a single peak of activity in the network. The manuscript, while explaining the dynamics of the system, did not discuss mathematical details that would govern the system.

From this point on, models used the same attractor based network to simulate path integrator characteristics of head direction cell systems. Models differed in underlying details, such as the neural components used in the system or the synaptic connections between these components. Another difference was the method of implementation used.

Blair \& and Sharp preferred to use the NEURON simulator [41] to provide a simulation of head direction cells [35] that included anticipatory firing characteristics that they had earlier discovered[35]. The NEURON simulator also permitted much lower level modelling where the researchers could simulate inhibitory and excitatory synapses between neuron sets that they mapped to brain regions based on anatomical data.

Redish et al.[42] proposed a coupled attractor model of head direction cells. The postsubiculum and anterior dorsal nucleus were both modelled as attractors and synaptic connections set up in such a way that the hill of activity in the attractor signifying the anterior dorsal nucleus always lead the other by a certain amount.

Zhang put forward an analytical model where he used quick changes in synaptic weights to generate the required attractor characteristics [43]. 


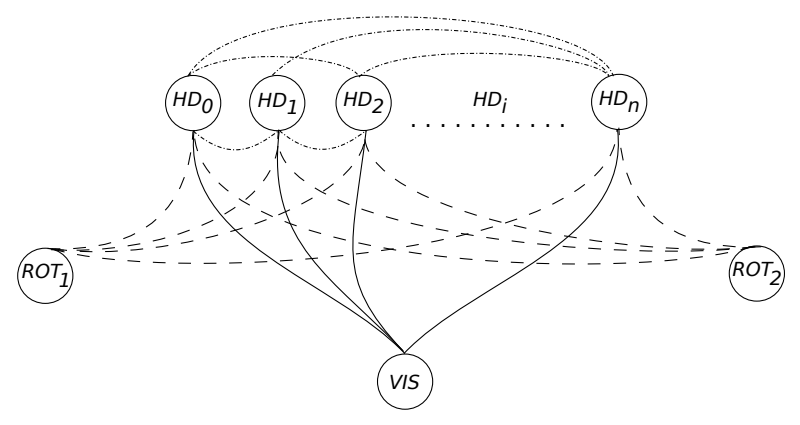

Fig. 1: The head direction cell set schematic.

All of the above mentioned models used synaptic weights that were predefined. Stringer et al. proposed a system based on Hebbian learning to explain how the synapses may be set up by self organization[14]. They only applied the method to model a path integrator system. Kyriacou extended the model to include kinesthetic inputs[44]: he implemented the model on a robot and used an omnidirectional camera to simulate visual inputs in the system. In another work, Kyriacou documents how a evolutionary algorithm[45] may be used to ascertain the parameters that controlled the dynamics of such a network[46].

Other models include but are not limited to: Arleo \& Gerstner's implementation on the mobile Khepera robot 47]; Song \& Wang's implementation that used a lower level spiking neuron architecture [48]; Degris and colleagues' implementation of Song \& Wang's work on to the Pekee robot as part of the Psikharpax project[49]; Goodridge \& Touretzky's model of the anticipatory characteristics of head direction cells that did not use an attractor network following observations that GABA containing interneurons that are necessary for attractor formation were absent in the anterior dorsal nucleus [38]; and Zeidman \& Bullinaria's extension to Goodridge \& Touretzky's model to include optic flow information[50].

\section{Methods: The MOdeL}

\section{A. Structure}

Our model is based on the self organizing model proposed by Stringer et al.[14]. Their original model presented a biologically plausible method of setting up synaptic weights in the head direction cell system, via Hebbian learning[51]. It only applied the method to the path integrator system of head direction cells and did not discuss visual inputs and landmark navigation. We've incorporated vision cells into their model in an attempt to extend it to project both vestibular and visual inputs on the head direction cell set as has been found.

The head direction cell system here makes use of three sets of neurons as shown in the Figure 1. The head direction cell set is a fully connected recurrent network, i.e., each neuron $H D_{i}$ is connected to every other neuron $H D_{j}$ via synapses, the weights of which are denoted as $w_{i j}^{H D}$. Here, $H D_{i}$ and $H D_{j}$ are the pre-synaptic and post-synaptic neurons respectively. The head direction cells, therefore,

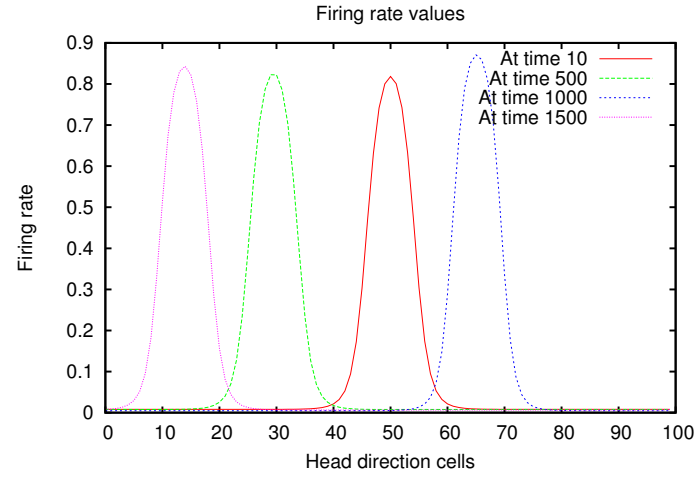

Fig. 2: Firing rates during test run.

form a one dimensional circular array. While cells with adjacent preferred directions appear next to each other in our implementation (and most models), this is not how head direction cells are found in the brain. In fact, as long as the synaptic connections are set up appropriately, the location of neurons is irrelevant to the functioning of the system.

Rotation cells $R O T_{k}$ are also connected to each head direction cell via synapses denoted $w_{i j k}^{H D_{-} R O T}$. Here, these synapses are not simply synapses between a singular head direction cell and a rotation cell. Rather, they're effective synapses between the pre-synaptic neuron $H D_{i}$, the postsynaptic neuron $H D_{j}$ and the rotation cell $R O T_{k}$. The two rotation cells fire depending upon clockwise and anticlockwise rotation inputs respectively as shown in Figure 5

The visual cell set represents an abstraction of the visual processing system and each visual cell $V I S_{l}$ is connected to every head direction cell $H D_{i}$ via synapses $w_{i l}^{H D_{-} V I S}$.

\section{B. Dynamics}

Our model is a firing rate based model, which provides a level of detail that is sufficient for our system. The activation of each head direction cell $H D_{i}$ is given by:

$$
\begin{aligned}
\tau \frac{\mathrm{d} h_{i}^{H D}(t)}{\mathrm{d} t}=-h_{i}^{H D}(t) & +\frac{\phi_{0}}{C^{H D}} \sum_{j}\left(w_{i j}^{R C}-w^{I N H}\right) r_{j}^{H D}(t) \\
& +\frac{\phi_{1}}{C^{H D \times R O T}} \sum_{j k}\left(w_{i j k}^{R O T} r_{j}^{H D} r_{k}^{R O T}\right) \\
& +\frac{\phi_{2}}{C^{H D \times V I S}} \sum_{j l}\left(w_{j l}^{V I S} r_{j}^{H D} r_{l}^{V I S}\right)
\end{aligned}
$$

Here, $\tau$ is the time constant while $\phi_{0}, \phi_{1}, \phi_{2}, C^{H D}$, $C^{H D \times R O T}, C^{H D \times V I S}$ and $w^{I N H}$ are tunable parameters. These parameters control the effect the respective inputs have on the head direction cell attractor. $w^{I N H}$ represents global inhibition that the GABAergic interneurons exert on the system. The combination of local excitation of head direction cells and the global inhibition gives the system continuous attractor characteristics. 
The firing rate of each head direction neuron is a sigmoid function of its activation:

$$
r_{i}^{H D}(t)=f\left(h_{i}^{H D}(t)\right)=\frac{1}{1+e^{-2 \beta\left(h_{i}^{H D}(t)-\alpha\right)}}
$$

where $\alpha$ and $\beta$ are constants. Figure 2 shows firing rate profiles exhibited by the head direction cell set during a run at different times. Due to the regular learning employed in this implementation, the firing rates of all head direction cells are similar. This isn't the case in biology, where the firing rates of head direction cells vary from one another.

The synapses between all neuron sets are set up using Hebbian learning:

$$
\Delta w=k \cdot\left(r^{\text {post }} * r^{\text {pre }}\right)
$$

Here, $\Delta w$ is the change in synaptic weight. $k$ is the learning rate of the synapse. $r^{\text {pre }}$ and $r^{\text {post }}$ are the firing rates of the pre-synaptic and post-synaptic neurons respectively. This learning rule does not, however, include synaptic depression, or bounding of synaptic weights. We use a competition based normalization rule to bound our synapses:

$$
\hat{w}=\frac{w}{|w|}
$$

Here $|w|$ is the norm of the $w$ matrix and $\hat{w}$ is the normalized synaptic weight. It is worth noting that the above normalization departs from the Hebbian learning requirement of locality[52],i.e., the synapse between two neurons should only be modified by their behaviour. We briefly discuss other formulations of Hebbian learning in section IV

\section{EXPERIMENTAL PROCEDURE AND RESUlTS}

We implemented the model based on the ROS(Robot operating system)[15] platform which provides support for a number of robots, including the PR2. ROS provides underlying tools that enable us to develop offline using simulators and then move the code as-is on to the robot. For development and testing, we collected data bags from the IMU sensors of the PR2 robot to run our simulations. We used a hundred head direction cells to cover the $360^{\circ}$ direction space. We used two rotation cells, one each for clockwise and anti clockwise rotation, and a single visual cell (Figure 11. The values of constants used in our implementation are given in table [.

The system runs in three phases:

\section{A. Setting up of synaptic weights to appropriate values}

During this first phase, we set up the synaptic weights in the network to their appropriate values. The network is initialized with all synaptic weights as zero, implying that no learning or association has taken place between the sets of neurons. In order to set up both the internal head direction cell synapses $w_{i j}^{H D}$ and the effective rotation synapses $w_{i j k}^{R O T}$, we simulate rotation in the system in both clockwise and anti-clockwise directions. Each head direction cell is assigned a preferred direction such that the set encompasses

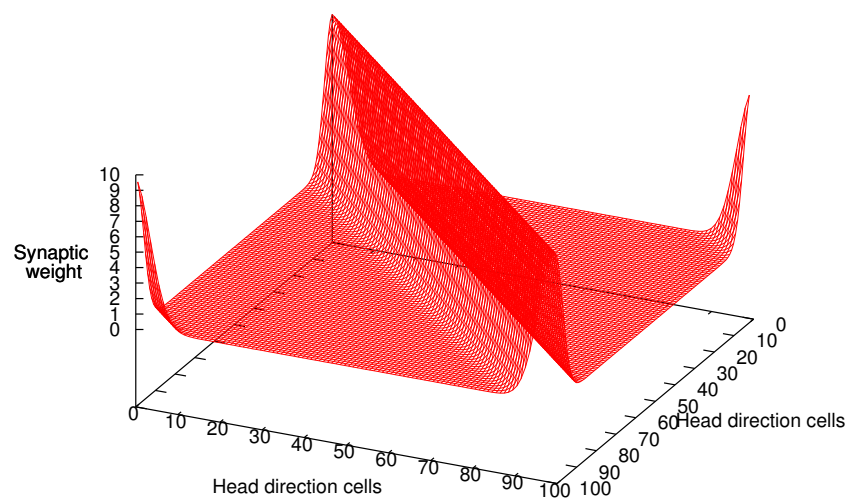

Fig. 3: $w_{i j}^{H D}$ values after training.

the complete $360^{\circ}$ range. As done previously in literature, we model the head direction cell firing profile as a Gaussian:

$$
r_{i}^{H D}=\exp \left(-\frac{1+\Delta S^{2}}{2 \sigma^{H D^{2}}}\right)
$$

$\Delta S$ is the angular distance between the current head direction and the head direction cell's preferred direction:

$$
\Delta S=\min (|x|,|360-x|)
$$

where, for each neuron $H D_{i}$ with preferred direction $x_{i}^{\text {preferred }}$, for a head direction $\theta$

$$
x=\theta-x_{i}^{\text {preferred }}
$$

$\sigma^{H D}$ is a constant that controls the width of the Gaussian profile, and consequently, controls the angular width that a head direction cell is active in.

In order to calibrate the network's synapses, one of the rotation cells is activated, simulating clockwise or anticlockwise rotation. Simultaneously, the firing rate profile, as obtained by equation (5), is simulated in the head direction cell and is moved to represent the change in heading of the agent. The synapses are modified as per the Hebbian learning rule discussed in section $\amalg$

Figure 3 shows the synaptic weights between head direction cells, $w_{i j}^{H D}$, before normalization. Note that as a result of Hebbian learning from the simulated Gaussian firing rate profiles, cells with preferred directions near each other have stronger synaptic connections than cells that are far apart, as is expected.

\section{B. Initializing the network to an initial direction}

Once the synaptic weights are set up appropriately, a packet of activity must be stimulated in the attractor. This packet of activity is the initial or reference heading of the agent. An initial packet of activity is forced on the system by projecting the required profile on to the head direction cell attractor from the visual input for a short period of time. The 


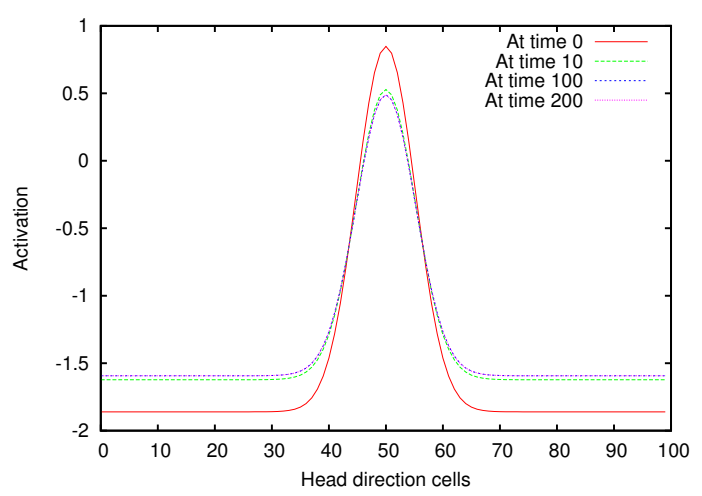

Fig. 4: Stabilization of initial activity packet

TABLE I: Constants used in the implementation

\begin{tabular}{c|c}
\hline Number of head direction cells & 100 \\
\hline Number of rotation cells & 2 \\
\hline Number of vision cells & 1 \\
\hline$\alpha$ & 1.5 \\
\hline$\beta$ & 3 \\
\hline$\phi_{0}$ & 100 \\
\hline$\phi_{1}$ & 200 \\
\hline$\phi_{2}$ & 100 \\
\hline$C^{H D}$ & 100 \\
\hline$C^{H D \times R O T}$ & 200 \\
\hline$C^{H D \times V I S}$ & 100 \\
\hline$k$ & 1 \\
\hline$w^{I N H}$ & 0.2 \\
\hline$\sigma^{H D}$ & 10 \\
\hline
\end{tabular}

firing of the vision cell can be simulated by simply setting its firing rate to the maximum value, 1 , in equation (1):

$$
r_{1}^{V I S}=1
$$

In order to set the initial direction to the preferred direction of head direction cell $i$, we simply set the synapses between the vision cell and head direction cells to the synaptic weight between head direction cell $i$ and every other head direction cell.

$$
w_{1, j}^{V I S}=w_{i j}^{H D}
$$

During our experiments, we observed that forcing an initial packet of activity did not guarantee a functional system. The attractor should maintain the packet of activity in the absence of external inputs. The parameters $\phi_{0}$ and $w^{I N H}$ that effect the recurrent behaviour of the network must be fine tuned to ensure that the activity packet stabilizes as shown in figure 4 , the other outcome being the activity packet flattening out ${ }^{\top}$

The continuous attractor, if set up properly, permits the packet of activity to lie in a state of neutral equilibrium, like a ball lying on a perfectly horizontal table surface.

\footnotetext{
${ }^{1}$ We are most grateful to Dr. Simon Stringer for his input on this subject.
}

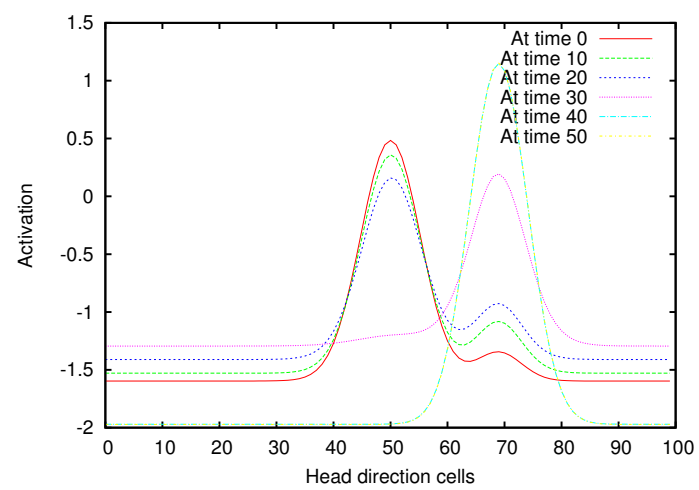

Fig. 6: Correcting drift using a single visual cue

\section{Running the system with angular velocity data}

Figure 5 shows the behaviour of the network when tested with angular velocity data. The firing rates of the two rotation cells are a linear function of the angular velocity inputs. The figure shows that the head direction indeed responds to angular velocity inputs. Of special interest is the graph's behaviour at time $=1300$ which shows circular nature of the network. The agent continues to change its head direction and seamlessly moves from $360^{\circ}$ to $0^{\circ}$. We tested the system with several bags of data and observed encouraging results.

The rate at which the head direction firing profile moves depends on the strength of the rotation neurons' projections on it. In the current configuration, the system has not been optimised to correctly map the rotations of the agent, i.e., the movement of the head direction activity profile does not reflect the true rotation of the agent in the world frame. This isn't because it cannot be done: the accuracy can be improved fine tuning the value of $\phi_{1}$ which controls the strength of the rotation neurons' projections on to the head direction cells. However, as we briefly discuss in the next sub section, our current work focusses on associating head direction cells to salient features in the environment for landmark navigation, and since this will function as a drift correction mechanism in itself, we've permitted the system to drift and have put off optimization of these constants.

\section{Correcting drift using salient visual cues: preliminary tests}

Figure 6 shows the results of a simulation where the head direction cell set is assumed to incur drift and, a projection from the visual feature cell, which would be caused by the agent observing a familiar feature, is used to correct this drift. We discuss our ideas on using multiple visual features in the next section.

\section{DISCUSSION}

Researchers have attempted to implement bio-inspired navigation systems in the past, although the level of inspiration and the extent of implementation has varied. The Psikharpax project [53], [54], for example, attempted to create an artificial rat and implemented head direction and place 

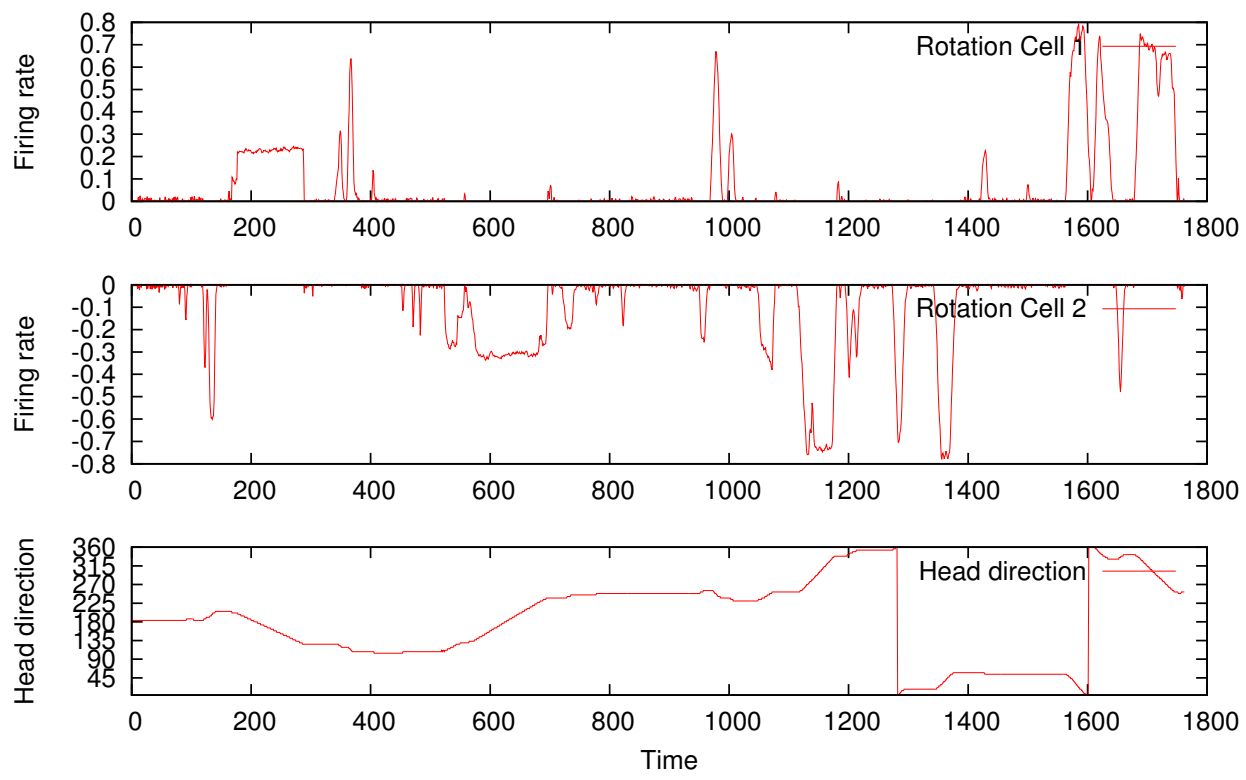

Fig. 5: Test runs with angular velocity data from the PR2 robot

cells as its basis for navigation. RatSLAM[55], on the other hand, uses similar "pose cells" to propose a solution to the SLAM problem. (For more examples, see Franz \& Mallot's review[1].)

Bio-mimetic navigation provides two closely related research areas. The first is to improve our understanding of biological navigation. This is done at different levels, for example, by via behavioural studies or neuron recordings. The second, computational modelling, serves as a tool to verify collected information and proposed theories, while providing alternative navigation systems that can, in the future, be used in robotics. Even though bio-mimetic systems are not yet considered mature enough for use in task oriented robotics ahead of classical navigation techniques, it is accepted that even smaller animals such as ants and rats possess navigational capabilities that are superior to classical robotic navigation techniques. The complexity of the underlying neural systems is a primary factor that makes bio-mimetic systems difficult to implement.

While the complexity presented by neural processing is difficult to simulate in its entirety, systems that achieve a high level of similarity can be designed. The model of the head direction system implemented here, for example, deviates from known information on head direction cells in certain aspects but does still sufficiently carry out its intended function.

\section{Challenges and future work}

The implementation of the path integrator half of the head direction cell system is the starting point in our attempt to develop a bio-mimetic navigation system for the PR2 robot using the ROS platform. The information this provides is not yet sufficient to carry out actual navigation. In the absence of a set of salient visual cues, the system will continue to drift as it runs, like any other INS[56]. An INS is generally coupled with other input sources, such as GPS[57], that reset the accumulated drift at regular intervals. The head direction system can similarly correct drift by detecting familiar visual features in the environment. Our next goal, is therefore, to associate the head direction cell system to environmental cues that will reset the head direction system to its associated direction whenever they are observed. Since the system will associate with a set of cues, it will capable of approaching these cues. The agent will be able to carry out the local navigation strategies: search, direction-following, aiming and guidance, as enumerated by Franz \& Mallot[1].

A further goal of ours is to implement a model of place cells that will hold location information about the agent. Place cells follow a system similar to head direction cells. They too carry out path integrator and associate to visual cues in the environment. Implementing a coupled place and head direction cell model will provide both location and heading information to the agent along with some information on visual features that they associate with. This will further improve the landmark navigation capabilities of the system.

An important part of the model is the Hebbian learning rule mentioned in equation (3). The formula that we've used currently is the simplest mathematical formulation of a Hebbian synaptic modification rule. As is visible, this rule does not provide for synaptic saturation. As long as the presynaptic and post-synaptic neurons fire simultaneously, the synapse between them will continue to strengthen. While this formulation covers strengthening of synapses by long term potentiation (LTP) [58], [59], it does not implement the 
flip side: long term depression (LTD) [60]. Just as LTP causes strengthening of synapses when presynaptic and postsynaptic neurons fire nearly simultaneously, LTD causes weakening of synapses if such simultaneous firing does not occur. The inability of the learning rule to provide for synaptic saturation and weakening makes it less biologically plausible. It also makes the implementation more difficult: if the synapses do not saturate at a known value, it is difficult to use constant values for parameters that control the projection of inputs on to head direction cells: $\phi_{0}, \phi_{1}, \phi_{2}$. During our simulations, we discovered that unbounded synapses constitute one of the cases where the projections on the head direction cells increase to such an extent that all neurons begin to fire maximally, making the network lose its peak, thus no longer providing information about the current heading.

A number of formulations of the Hebbian rule have been proposed in literature [61], [62]. Such rules incorporate modifications to provide for LTD and synaptic saturation. One such modification is to normalize the synaptic weights, as shown in equation (4). Normalization is a competition based method: if synaptic efficacy increases, it must be at the expense of other synapses[52]. Other formulations include gating of the synaptic changes by either presynaptic or postsynaptic activity. While we did attempt to use gated rules with saturation in our model, we were unable to find a set of parameters that provided the required dynamics. Since the recurrent synapses between the head direction cells and the synapses between rotation cells and head direction cells remain largely unchanged during the running of the system, we decided to use the normalization rule and fix the synaptic weights after initial calibration of the system. For association with visual features, however, the formulation that is used to modify synapses between the head direction and visual cells will need to incorporate weakening of synapses via LTD so that the system can disassociate with (forget) features that are no longer present in the environment over a period of time.

The inclusion of multiple visual features presents a challenge also. In order for the system to associate with more than one feature, our implementation of visual cells should be able to differentiate between features so that they can each be encoded uniquely by our population of vision cells. We are yet to decide on a method to obtain this since modelling the biological visual system may be too complex for our purposes.

\section{SUMmary AND CONCLUSION}

An implementation of a model of the head direction cell system on the ROS platform was detailed in this paper. The implementation integrates angular velocity inputs using neural mechanisms to track the orientation of the robot. Certain extensions to the original model required to enable integration of visual inputs were also discussed. Visual inputs provide sensory information that is used to correct drift in the path integrator mechanism. The anchoring of head direction cells to visual landmarks makes it possible for the system to also provide a feature based navigation method. While a landmark navigation system will be limited to navigating between locations that are visible to the agent, they can be coupled with more complex map based methods to provide a layered navigation system. Various challenges and their tentative solutions were discussed as work to be done in the future to follow up the path integrator system.

Biological navigation follows a layered approach. The navigation technique employed depends on the task to be completed by the organism. For example, it is inefficient to use metric mapped navigation to approach a goal state that is visible to an agent. In such cases, simple navigation techniques such as piloting or landmark navigation would be quicker and more efficient. With this implementation of the path integrator dynamics of a head direction cell ensemble, we move closer to developing a landmark based navigation system as the simplest component of a layered bio-mimetic system for the PR2 robot.

\section{REFERENCES}

[1] M. O. Franz and H. A. Mallot, "Biomimetic robot navigation," Robotics and autonomous systems, vol. 30, no. 1, pp. 133-153, 2000.

[2] O. Trullier, S. I. Wiener, A. Berthoz, and J.-A. Meyer, "Biologically based artificial navigation systems: Review and prospects," Progress in neurobiology, vol. 51, no. 5, pp. 483-544, 1997.

[3] R. U. Muller, J. B. Ranck, and J. S. Taube, "Head direction cells: properties and functional significance," Current opinion in neurobiology, vol. 6, no. 2, pp. 196-206, 1996.

[4] J. Ranck Jr, "Head direction cells in the deep cell layer of dorsal presubiculum in freely moving rats," in Society for Neuroscience Abstracts, vol. 10, 1984.

[5] J. O'Keefe, "Place units in the hippocampus of the freely moving rat," Experimental neurology, vol. 51, no. 1, pp. 78-109, 1976.

[6] T. Hafting, M. Fyhn, S. Molden, M.-B. Moser, and E. I. Moser, "Microstructure of a spatial map in the entorhinal cortex," Nature, vol. 436, no. 7052, pp. 801-806, 2005.

[7] R. U. Muller, M. Stead, and J. Pach, "The hippocampus as a cognitive graph." The Journal of general physiology, vol. 107, no. 6, pp. 663694, 1996.

[8] B. L. McNaughton, F. P. Battaglia, O. Jensen, E. I. Moser, and M.-B. Moser, "Path integration and the neural basis of the'cognitive map'," Nature Reviews Neuroscience, vol. 7, no. 8, pp. 663-678, 2006.

[9] O. Trullier and J.-A. Meyer, "Animat navigation using a cognitive graph," Biological Cybernetics, vol. 83, no. 3, pp. 271-285, 2000.

[10] J. O'Keefe, “The hippocampal cognitive map and navigational strategies." 1991.

[11] L. Nadel and L. MacDonald, "Hippocampus: Cognitive map or working memory?" Behavioral and neural biology, vol. 29, no. 3, pp. 405409, 1980.

[12] J. O'Keefe and L. Nadel, The hippocampus as a cognitive map. Clarendon Press Oxford, 1978, vol. 3.

[13] B. Barshan and H. F. Durrant-Whyte, "Inertial navigation systems for mobile robots," Robotics and Automation, IEEE Transactions on, vol. 11, no. 3, pp. 328-342, 1995.

[14] S. Stringer, T. Trappenberg, E. Rolls, and I. E. T. Araujo, "Selforganizing continuous attractor networks and path integration: onedimensional models of head direction cells," Network: Computation in Neural Systems, vol. 13, no. 2, pp. 217-242, 2002.

[15] M. Quigley, K. Conley, B. Gerkey, J. Faust, T. Foote, J. Leibs, R. Wheeler, and A. Y. Ng, "Ros: an open-source robot operating system," in ICRA workshop on open source software, vol. 3, 2009.

[16] J. S. Taube, "Head direction cells and the neurophysiological basis for a sense of direction," Progress in neurobiology, vol. 55, no. 3, pp. 225-256, 1998.

[17] S. I. Wiener and J. S. Taube, Head Direction Cells and the Neural Mechanisms of Spatial Orientation (Bradford Books). The MIT press, 2005.

[18] J. S. Taube, "The head direction signal: origins and sensory-motor integration,” Аnnu. Rev. Neurosci., vol. 30, pp. 181-207, 2007. 
[19] _ - "Head direction cells recorded in the anterior thalamic nuclei of freely moving rats," The journal of neuroscience, vol. 15, no. 1, pp. 70-86, 1995.

[20] S. Mizumori and J. Williams, "Directionally selective mnemonic properties of neurons in the lateral dorsal nucleus of the thalamus of rats," The journal of neuroscience, vol. 13, no. 9, pp. 4015-4028, 1993.

[21] L. L. Chen, L.-H. Lin, E. J. Green, C. A. Barnes, and B. L. McNaughton, "Head-direction cells in the rat posterior cortex," Experimental Brain Research, vol. 101, no. 1, pp. 8-23, 1994.

[22] C. L. Leonhard, R. W. Stackman, and J. Taube, "Head direction cells recorded from the lateral mammillary nuclei in rats," Society for Neuroscience Abstracts, vol. 22, 1996.

[23] S. I. Wiener, "Spatial and behavioral correlates of striatal neurons in rats performing a self-initiated navigation task," The journal of neuroscience, vol. 13, no. 9, pp. 3802-3817, 1993.

[24] S. Mizumori and B. Cooper, "Spatial representations of dorsal caudate neurons of freely-behaving rats," in Society for Neuroscience Abstracts, vol. 21,1995 , p. 1929.

[25] R. G. Robertson, E. T. Rolls, P. Georges-Franois, S. Panzeri et al., "Head direction cells in the primate pre-subiculum," Hippocampus, vol. 9, no. 3, pp. 206-219, 1999.

[26] J. S. Taube, R. U. Muller, and J. B. Ranck Jr, "Head-direction cells recorded from the postsubiculum in freely moving rats. ii. effects of environmental manipulations," The journal of neuroscience, vol. 10 , no. 2, pp. 436-447, 1990.

[27] M. B. Zugaro, A. Arleo, A. Berthoz, and S. I. Wiener, "Rapid spatial reorientation and head direction cells," The journal of neuroscience, vol. 23, no. 8, pp. 3478-3482, 2003.

[28] M. B. Zugaro, A. Berthoz, and S. I. Wiener, "Background, but not foreground, spatial cues are taken as references for head direction responses by rat anterodorsal thalamus neurons." The Journal of neuroscience: the official journal of the Society for Neuroscience, vol. 21, no. 14, p. RC154, 2001.

[29] H. T. Blair and P. E. Sharp, "Visual and vestibular influences on head-direction cells in the anterior thalamus of the rat." Behavioral neuroscience, vol. 110, no. 4, p. 643, 1996.

[30] J. B. Kelly and S. J. Glazier, "Auditory cortex lesions and discrimination of spatial location by the rat," Brain Research, vol. 145, no. 2, pp. 315-321, 1978

[31] J. P. Goodridge, P. A. Dudchenko, K. A. Worboys, E. J. Golob, and J. S. Taube, "Cue control and head direction cells." Behavioral neuroscience, vol. 112 , no. 4, p. 749, 1998

[32] J. S. Taube, R. U. Muller, and J. B. Ranck Jr, "Head-direction cells recorded from the postsubiculum in freely moving rats. i. description and quantitative analysis," The journal of neuroscience, vol. 10, no. 2, pp. 420-435, 1990

[33] M. B. Zugaro, E. Tabuchi, C. Fouquier, A. Berthoz, and S. I. Wiener, "Active locomotion increases peak firing rates of anterodorsal thalamic head direction cells," Journal of neurophysiology, vol. 86, no. 2, pp. 692-702, 2001

[34] J. S. Taube and R. U. Muller, "Comparisons of head direction cell activity in the postsubiculum and anterior thalamus of freely moving rats," Hippocampus, vol. 8, no. 2, pp. 87-108, 1998. [Online]. Available: http://dx.doi.org/10.1002/(SICI)1098-1063(1998) 8:2 $\langle 87::$ AID-HIPO1 $\rangle$ 3.0.CO $2-4$

[35] H. T. Blair and P. E. Sharp, "Anticipatory head direction signals in anterior thalamus: evidence for a thalamocortical circuit that integrates angular head motion to compute head direction," The journal of neuroscience, vol. 15 , no. 9, pp. 6260-6270, 1995 .

[36] H. T. Blair, B. W. Lipscomb, and P. E. Sharp, "Anticipatory time intervals of head-direction cells in the anterior thalamus of the rat: implications for path integration in the head-direction circuit," Journal of neurophysiology, vol. 78, no. 1, pp. 145-159, 1997.

[37] M. Bentivoglio, K. Kultas-Ilinsky, and I. Ilinsky, "Limbic thalamus: structure, intrinsic organization, and connections," Neurobiology of cingulate cortex and limbic thalamus: A comprehensive handbook, pp. 71-122, 1993.

[38] J. P. Goodridge and D. S. Touretzky, "Modeling attractor deformation in the rodent head-direction system," Journal of neurophysiology, vol. 83, no. 6, pp. 3402-3410, 2000.

[39] B. L. McNaughton, L. Chen, and E. Markus, "“'dead reckoning”, landmark learning, and the sense of direction: A neurophysiological and computational hypothesis," Journal of Cognitive Neuroscience, vol. 3, no. 2, pp. 190-202, 1991.
[40] W. E. Skaggs, J. J. Knierim, H. S. Kudrimoti, and B. L. McNaughton, "A model of the neural basis of the rat's sense of direction," in Advances in Neural Information Processing Systems 7: Proceedings of the 1994 Conference, vol. 7. MIT Press, 1995, p. 173.

[41] M. L. Hines and N. T. Carnevale, "The neuron simulation environment," Neural Computation, vol. 9, no. 6, pp. 1179-1209, 1997.

[42] D. A. Redish, A. N. Elga, and D. S. Touretzky, "A coupled attractor model of the rodent head direction system," Network: Computation in Neural Systems, vol. 7, no. 4, pp. 671-685, 1996. [Online]. Available: http://informahealthcare.com/doi/abs/10.1088/0954-898X_7_4_004

[43] K. Zhang, "Representation of spatial orientation by the intrinsic dynamics of the head-direction cell ensemble: a theory," The journal of neuroscience, vol. 16, no. 6, pp. 2112-2126, 1996

[44] T. Kyriacou, "An implementation of a biologically inspired model of head direction cells on a robot," in Towards Autonomous Robotic Systems, ser. Lecture Notes in Computer Science, R. Gro, L. Alboul, C. Melhuish, M. Witkowski, T. Prescott, and J. Penders, Eds. Springer Berlin Heidelberg, 2011, vol. 6856, p. 66. [Online]. Available: http://dx.doi.org/10.1007/978-3-642-23232-9_7

[45] H.-G. Beyer and H.-P. Schwefel, "Evolution strategies-a comprehensive introduction," Natural computing, vol. 1, no. 1, pp. 3-52, 2002.

[46] T. Kyriacou, "Using an evolutionary algorithm to determine the parameters of a biologically inspired model of head direction cells," Journal of computational neuroscience, vol. 32, no. 2, pp. 281-295, 2012.

[47] A. Arleo and W. Gerstner, "Spatial cognition and neuro-mimetic navigation: A model of hippocampal place cell activity," Biological Cybernetics, vol. 83, no. 3, pp. 287-299, 2000.

[48] P. Song and X.-J. Wang, "Angular path integration by moving hill of activity: a spiking neuron model without recurrent excitation of the head-direction system," The journal of neuroscience, vol. 25, no. 4, pp. 1002-1014, 2005.

[49] T. Degris, L. Lacheze, C. Boucheny, and A. Arleo, "A spiking neuron model of head-direction cells for robot orientation," in Proceedings of the Eighth Int. Conf. on the Simulation of Adaptive Behavior, from Animals to Animats, 2004, pp. 255-263.

[50] P. Zeidman and J. A. Bullinaria, "Neural models of head-direction cells," From associations to rules: Connectionist models of behavior and cognition, pp. 165-177, 2008.

[51] D. Hebb, "The organization of behavior: A neuropsychological theory," 1949.

[52] W. Gerstner and W. M. Kistler, Spiking neuron models: Single neurons, populations, plasticity. Cambridge university press, 2002.

[53] J.-A. Meyer, A. Guillot, B. Girard, M. Khamassi, P. Pirim, and A. Berthoz, "The psikharpax project: Towards building an artificial rat," Robotics and autonomous systems, vol. 50, no. 4, pp. 211-223, 2005.

[54] J.-A. Meyer, A. Guillot, P. Pirim, A. Berthoz, and F. BEV, "Psikharpax: An autonomous and adaptive artificial rat," in Proceedings of ISR2004, the 35th International Symposium on Robotics, Paris, 2004.

[55] M. J. Milford, G. F. Wyeth, and D. Prasser, "Ratslam: a hippocampal model for simultaneous localization and mapping," in Robotics and Automation, 2004. Proceedings. ICRA'04. 2004 IEEE International Conference on, vol. 1. Ieee, 2004, pp. 403-408.

[56] S. Sukkarieh, "Low cost, high integrity, aided inertial navigation systems for autonomous land vehicles," Ph.D. dissertation, The University of Sydney, 2000.

[57] S. Panzieri, F. Pascucci, and G. Ulivi, "An outdoor navigation system using gps and inertial platform," Mechatronics, IEEE/ASME Transactions on, vol. 7, no. 2, pp. 134-142, 2002.

[58] T. V. Bliss and T. Lmo, "Long-lasting potentiation of synaptic transmission in the dentate area of the anaesthetized rabbit following stimulation of the perforant path," The Journal of physiology, vol. 232, no. 2, pp. 331-356, 1973.

[59] T. Teyler and P. DiScenna, "Long-term potentiation," Annual review of neuroscience, vol. 10, no. 1, pp. 131-161, 1987.

[60] M. Ito, "Long-term depression," Annual review of neuroscience, vol. 12 , no. 1 , pp. $85-102,1989$.

[61] W. Gerstner and W. M. Kistler, "Mathematical formulations of hebbian learning," Biological Cybernetics, vol. 87, no. 5-6, pp. 404-415, 2002.

[62] K. D. Miller and D. J. MacKay, "The role of constraints in hebbian learning," Neural Computation, vol. 6, no. 1, pp. 100-126, 1994. 